\title{
PHOENIX model chromospheres of mid- to late-type M dwarfs ${ }^{\star, \star \star, \star \star \star}$
}

\author{
B. Fuhrmeister, J. H. M. M. Schmitt, and P. H. Hauschildt
}

\author{
Hamburger Sternwarte, University of Hamburg, Gojenbergsweg 112, 21029 Hamburg, Germany \\ e-mail: bfuhrmeister@hs.uni-hamburg.de
}

Received 9 November 2004 / Accepted 28 April 2005

\begin{abstract}
We present semi-empirical model chromospheres computed with the atmosphere code PHOENIX. The models are designed to fit the observed spectra of five mid- to late-type $\mathrm{M}$ dwarfs. Next to hydrogen lines from the Balmer series we used various metal lines, e.g. from Fe I, for the comparison between data and models. Our computations show that an NLTE treatment of C, N, O impacts on the hydrogen line formation, while NLTE treatment of less abundant metals such as nickel influences the lines of the considered species itself. For our coolest models we investigated also the influence of dust on the chromospheres and found that dust increases the emission line flux.

Moreover we present an (electronically published) emission line list for the spectral range of 3100 to 3900 and 4700 to $6800 \AA$ for a set of $21 \mathrm{M}$ dwarfs and brown dwarfs. The line list includes the detection of the Na I D lines in emission for a L3 dwarf.
\end{abstract}

Key words. stars: activity - stars: late-type - stars: chromospheres

\section{Introduction}

Chromospheric activity as indicated, for example, by $\mathrm{H}_{\alpha}$ emission is frequently found in early-type $\mathrm{M}$ dwarfs, and ubiquitously in mid to late-type $\mathrm{M}$ dwarfs. There are indications that the $\mathrm{H}_{\alpha}$ emission during quiescence declines for very late-type M dwarfs and L dwarfs (Liebert et al. 2003; Gizis et al. 2000), although even brown dwarfs can show $\mathrm{H}_{\alpha}$ emission at least during flares. Since the heating mechanisms of chromospheres and coronae are poorly understood, we may hope to learn more about the observed emission lines via the construction of semiempirical chromospheres.

Semi-empirical modelling of the chromosphere of the Sun was carried out quite successfully by Vernazza et al. (1981), determining the temperature distribution versus the column mass $(m)$. Early models for M dwarfs were constructed by Cram \& Mullan (1979). More recently, Hawley \& Fisher (1992) constructed chromosphere and transition region (TR) models of different activity level including soft X-ray emission from the corona. An ansatz using a linear temperature rise vs. $\log m$ in the chromosphere and TR was used by Short $\&$ Doyle (1997) and related papers (e.g. Short \& Doyle 1998;

\footnotetext{
* Based on observations collected at the European Southern Observatory, Paranal, Chile, 68.D-0166A.

$\star \star$ Appendix A is only available in electronic form at http://www.edpsciences.org

$\star \star \star$ Table A.2 is only available in electronic form at the CDS via anonymous ftp to cdsarc.u-strasbg.fr $(130.79 .128 .5)$ or via http://cdsweb.u-strasbg.fr/cgi-bin/qcat? J/A+A/439/1137
}

Andretta et al. 1997). These investigators used the atmospheric code MULTI (Carlsson 1986), and in addition the atmospheric code PHOENIX (Hauschildt et al. 1999) to calculate background opacities. Falchi \& Mauas (1998) instead used the atmosphere code Pandora (Avrett \& Loeser 1984) and a nonlinear temperature versus $\log m$ distribution. The lines under consideration were usually the $\mathrm{Ly}_{\alpha}$ line, the $\mathrm{H}_{\alpha}$ line, the $\mathrm{Ca}$ II $\mathrm{H}$ and $\mathrm{K}$ lines and a few other metal lines. One problem with such models lies in the uniqueness of the description. In other words: can there be two different models producing the same line fluxes? Naturally this problem must be more severe if only few lines are used for adjusting the model.

With the advent of large telescopes like the VLT it is now possible to obtain high quality spectra in the (optical) near UV, where M dwarfs exhibit hundreds of chromospheric emission lines. Since most of these lines are Fe I and II lines, at least Fe has to be computed in NLTE in addition to $\mathrm{H}$ and $\mathrm{He}-$ an approach that has become possible in the last few years due to increasing computing power.

In this paper we present model chromospheres for mid-type $\mathrm{M}$ dwarfs during quiescent state adjusted via various lines in the wavelength range between 3600 and $6600 \AA$. Our paper is structured as follows: in Sect. 2 we describe the VLT data used for our analysis and the sample of M dwarfs. In Sect. 3 we deal with the model construction and describe the influence of various model parameters in Sect. 4. We present our best fit models for the individual stars in Sect. 5 and discuss several aspects of the models in Sect. 6. In the appendix a catalog of chromospheric emission lines is presented. 


\section{Observations and data analysis}

A set of $23 \mathrm{M}$ dwarf spectra was taken with UVES/VLT in visitor and in service mode between winter 2000 and March 2002. The original sample was designed for a search for the forbidden Fe XIII line at $3388 \AA$ and covers the whole M dwarf regime from M 3.5 plus a few $\mathrm{L}$ dwarfs known to show $\mathrm{H}_{\alpha}$ activity. All stars were selected for their high activity level. Two of the stars were double stars and since the spectra could not be disentangled, they were excluded from the analysis, and we ended up with 21 objects.

The five objects used in the modelling were selected to cover the $\mathrm{M}$ dwarf temperature regime with good $S / N$ ratios and without obvious flaring activity during the observations. The spectra were obtained in visitor mode with ESO's Kueyen telescope at Paranal equipped with the Ultraviolet-Visual Echelle Spectrograph (UVES) from March, 13th to 16th in 2002. The instrument was operated in dichroic mode, yielding 33 echelle orders in the blue arm (spectral coverage from 3030 to $3880 \AA$ ) and 39 orders in the red arm (spectral coverage from 4580 to $6680 \AA$ A). Therefore we cannot observe the lines from $\mathrm{H}_{3}$ up to $\mathrm{H}_{8}$ of the Balmer series, nor do we cover the $\mathrm{Ca}$ II $\mathrm{H}$ and $\mathrm{K}$ lines. The typical resolution of our spectra is $\sim 45000$, typical exposures lasted 5 to $20 \mathrm{~min}$. Unfortunately the $\mathrm{H}_{\alpha}$ line is saturated in all of our spectra for AD Leo, CN Leo and YZ CMi. On the other hand, the blue part of the spectrum is underexposed for LHS 3003 and partly for DX Cnc, and therefore was not used for the modelling.

All data were reduced using IRAF in a standard way. The wavelength calibration was carried out using thorium-argon spectra with an accuracy of $\sim 0.03 \AA$ in the blue arm and $\sim 0.05 \AA$ in the red arm (i.e., more than 90 percent of the residuals of the wavelength calibration are lower than this value; the same is found for the difference between measured and laboratory wavelength in the emission line measurements presented in the appendix). In addition to the UVES spectra there are photometric data from the UVES exposure meter. These data were actually taken for engineering purposes, and are therefore not flux calibrated. Still, these data were useful to assess whether the star was observed during quiescence or during a major flare. We used the photometer data to decide which spectrum was taken during quiescence and therefore can be used for the chromospheric modelling. If no flare occurred during the whole observation and the spectra seem to be quite stable we used the averaged spectrum to obtain a better $S / N$ (see Table 1).

We show two typical parts of the spectrum of CN Leo in Figs. 1 and 2 to point out the wealth of emission lines in the blue part of the spectrum. There are hundreds of emission lines originating in the chromosphere, all of which could in principle be used for the modelling. Since the lines belong to many different species we had to decide which species to use since not all of them could be calculated in NLTE if computation times are to remain reasonable (see Sect. 3). Identifications of the emission lines show that by far most of them are Fe I lines. For more detailed informations on the emission lines seen on the different stars we provide a catalog of emission line identification in the appendix.

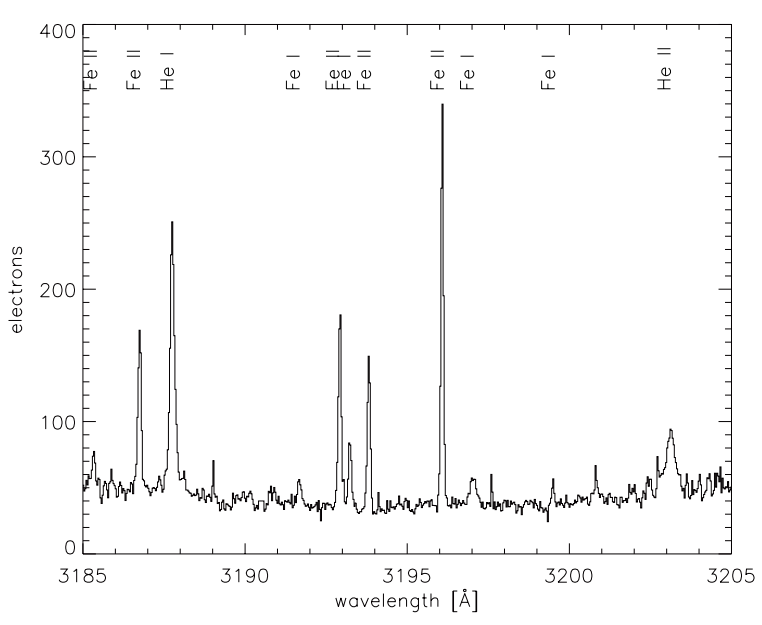

Fig. 1. A typical blue part of the spectrum of CN Leo around $3200 \AA$. The emission lines belong to Fe I, Fe II, He I and He II.

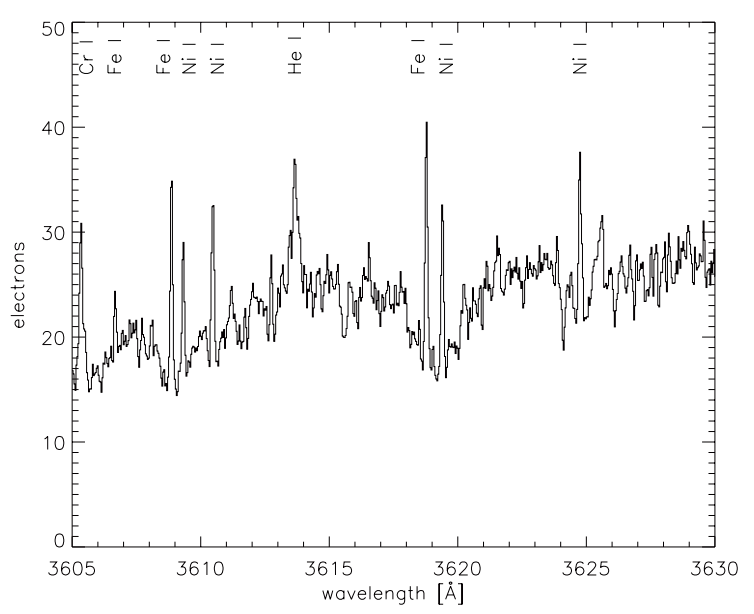

Fig. 2. Same as is Fig. 1 around $3600 \AA$ A. The emission lines belong to Ni I, Fe I, Cr I and He I.

\section{Model construction and calculation}

The model atmospheres consist of an underlying photosphere in radiative equilibrium, a chromosphere and part of the TR with a given temperature rise. All computations were carried out with the atmosphere code PHOENIX v13.7 (Hauschildt et al. 1999). For computations of $\mathrm{M}$ dwarfs chromosphere the proper NLTE treatment of the considered species and the background opacities are especially important (Andretta et al. 1997), both of which are provided by PHOENIX. Drawbacks in the chromospheric computations with PHOENIX are that no coronal flux is - yet - incorporated and that all lines are computed with the assumption of complete redistribution. Partial redistribution is especially important for the $\mathrm{Ca} \mathrm{H}$ and $\mathrm{K}$ lines and for $\mathrm{Ly}_{\alpha}$; therefore none of these lines has been used in the modelling. Another assumption generally made in semiempirical chromosphere modelling is hydrostatic and ionisation equilibrium throughout the atmosphere. This assumption has been challenged recently for the Sun (e.g. Carlsson \& Stein 2002), but there are no detailed hydrodynamic chromospheric simulations for $\mathrm{M}$ dwarfs available. 
Table 1. Parameters of the underlying photospheres for the model construction. From the literature we also cite $[\mathrm{A} / \mathrm{H}]$ given in brackets.

\begin{tabular}{cccccc}
\hline \hline Star & Other name & Spectrum used & $T_{\text {eff }}[\mathrm{K}]$ & $\log g$ & Literature \\
\hline AD Leo & GJ 388 & 2002-03-16 03:40:47 & 3200 & 4.5 & $3350,4.5(-0.75)^{1}$ \\
YZ CMi & GJ 285 & average & 3000 & 4.5 & $2925^{3}$ \\
CN Leo & GJ 406 & 2002-03-14 03:24:38 & 2900 & 5.5 & $2900,5.0(0.0)^{1}$ \\
DX Cnc & GJ 1111 & average & 2700 & 5.0 & $2850,5.25(+0.5)^{1} ; 2775^{3}$ \\
LHS 3003 & GJ 3877 & average & 2500 & 4.5 & $2400-2650^{2}$ \\
\hline
\end{tabular}

${ }^{1}$ Jones et al. (1996), ${ }^{2}$ Leggett et al. (2002), ${ }^{3}$ Mohanty \& Basri (2003).

\subsection{The underlying photospheres}

For the photospheres we used the best fit models to the red part of the UVES spectra which was determined by a $\chi^{2}$ technique. The model grid for the comparison ranged from $T_{\text {eff }}=$ $2300-3700 \mathrm{~K}$ and $\log g=4.0-5.5$ in steps of $\Delta T_{\text {eff }}=100 \mathrm{~K}$ and $\Delta \log g=0.5$. The photospheric $\log g$ and $T_{\text {eff }}$ parameters determined in that fashion are listed in Table 1. We used solar chemical composition for all modelling since little is known even for the photospheric abundances in M dwarfs. In principle an abundance analysis for the chromosphere is possible using the emission lines - provided the temperature structure for the star is known. Such a technique would be especially useful for the late-type $\mathrm{M}$ dwarfs where atomic photospheric lines are becoming rare.

\subsection{Construction of the chromospheres}

For the chromospheres we used the same ansatz as Short \& Doyle (1997), i.e., a linear temperature rise vs. $\log m$ with different gradients in the chromosphere and TR. For some models we deviated from the linear temperature rise in two different ways. For two stars we decided to alter the temperature structure in the lower chromosphere to obtain a better fit of the Na I D lines (see Fig. 4). This can be done without altering the flux in the other diagnostic lines too much and is based on the gradient of models that fitted the Na I D line better than the model that gives the best overall fit. In the other case we tried to compose the chromosphere of two linear segments, introducing a plateau in the upper chromosphere with the second linear part. This leads to significantly more flux in the Balmer lines and these models were all inferior to a single gradient temperature rise. However, the results of our modelling suggests, that deviations from the linear temperature rise may improve the fits, since different temperature gradients fitted different lines best. For this paper we decided to stick to the linear temperature rise, though and obtain an overall best fit. The top of the chromosphere is chosen at $8000 \mathrm{~K}$ for all models since for higher temperatures hydrogen is no efficient cooling agent any more (Ayres 1979).

\subsection{Construction of the TR}

The TR in our models extends up to $\log T=5.0$. In principle the gradient in the TR should be given by Spitzer conduction. Since conduction is balanced by the emerging flux from the TR, the gradient can be computed if the flux from the TR is known. For late type M dwarfs there are very few measurements for UV fluxes for temperatures below $\log T=5.0$. Therefore we decided to model the TR temperature gradient as a function of the logarithmic column mass $(\log m)$ semi-empirically as well, using the parameter $\log \frac{\mathrm{d} T}{\mathrm{~d} \log m}$. Balancing the conductive energy flux given by $\kappa_{0} T^{5 / 2} \frac{\mathrm{d} T}{\mathrm{~d} s}$ (e.g. Mihalas 1978) with the emerging radiation one obtains

$\frac{\mathrm{d}}{\mathrm{d} s}\left(\kappa_{0} T^{5 / 2} \frac{\mathrm{d} T}{\mathrm{~d} s}\right)=n^{2} P(T)$

with $P(T)$ denoting the cooling function of a hot radiating plasma, $T$ the temperature and $\kappa_{0} \approx 8.0 \times 10^{-7}$ in $\mathrm{cgs}$ units (Mihalas 1978). On neglecting the term involving second derivatives this leads to

$\frac{\mathrm{d} T}{\mathrm{~d} \log m}=m T^{-3 / 4} P(T)^{1 / 2} \cdot$ const.

Since $P(T) \sim T^{3 / 2}$ for $20000<T<100000$ and $m$ is about constant in the TR our ansatz $\log \frac{\mathrm{d} T}{\mathrm{~d} \log m}=$ const. is in good agreement with a conduction dominated energy transport. The gradients we find in the best fit models also agree with the gradients found by Jordan et al. (1987) for $\mathrm{G} / \mathrm{K}$ dwarfs in the 50000 to $100000 \mathrm{~K}$ regime inferred from the emission measure distribution.

\subsection{Treatment of turbulent pressure}

The chromosphere models are computed in hydrostatic equilibrium on a given column mass grid including convection and turbulent pressure. The treatment of the turbulent velocity $\xi$ and the resulting pressure is crucial for the models. We first used $\xi=2 \mathrm{~km} \mathrm{~s}^{-1}$ in the photosphere and a linear rise to $10 \mathrm{~km} \mathrm{~s}^{-1}$ at the top of the chromosphere continuing into the whole TR. However, the models with $T_{\min }$ at higher pressure do not converge for a linear turbulent velocity rise since it attains a significant fraction of the sound velocity or even becomes larger than the sound velocity in individual layers. Therefore the high pressure models are only possible for a description of the turbulent velocity as a fraction of the sound velocity. We chose $\xi$ as $0.5 v_{\text {sound }}$. For the same model $\xi=0.7 v_{\text {sound }}$ produces lower emission lines than $\xi=0.5 v_{\text {sound }}$ in the Balmer series and lower emission cores with self absorption for the Na I D lines. This is due to the lower electron pressure in the high turbulence velocity model as discussed by Jevremović et al. (2000). A turbulent velocity of $2 \mathrm{~km} \mathrm{~s}^{-1}$ is applied to all photospheric layers in all our models. 


\subsection{NLTE treatment}

Moreover we took into account NLTE effects for various species. The models presented here normally treat $\mathrm{H}, \mathrm{He}$, C I-III, N I-III, O I-III, Fe I-IV, Ti I-Ti II, Na I-IV and Mg I-III in NLTE. For some tests different NLTE sets of ions are chosen, which will be pointed out for these models. All NLTE computations take into account all levels from the Kurucz database (Kurucz \& Bell 1995) or from the CHIANTI database (Young et al. 2003) for C V to C VI, N VII and O V to O VIII and Fe VI to Fe VII. Since NLTE computations show that the level populations of most species and especially hydrogen are far from LTE we also took into account scattering for the LTE background lines, since not all lines can be computed in NLTE. The scattering of the background lines is treated by choosing the $\varepsilon$ parameter in the relation $S=\varepsilon B+(1-\varepsilon) J$, where $S$ is the source function, $B$ the Planck function and $\mathrm{J}$ the mean intensity. If no scattering is included, i.e. $\varepsilon=1.0$, we obtain unrealisticly high emission in the Balmer lines for moderate chromospheres and for stronger chromospheres even a Balmer jump and significant Balmer continuum, which is not observed in any of our spectra. Since the NLTE level population strongly deviates from the LTE level population in the chromosphere one would expect a lower value of $\varepsilon$. Therefore we lowered $\varepsilon$ to 0.5 and to 0.1 . The latter value gave no Balmer jump and realistic Balmer line emission. Therefore we used $\varepsilon=0.1$ for all the model computations. Since the $\varepsilon$ grid is rather coarse, the uncertainties in this parameter are large. The model spectra needed clearly $\varepsilon<0.5$ to avoid producing a Balmer jump, but for $\varepsilon<0.5$ it should be possible to balance the emergent emission with a TR onset at lower column mass. For the best fit model of AD Leo we computed also a model with $\varepsilon=0.2$ which would lead to a lowering of the column mass at the onset of the TR of about 0.1 dex.

\subsection{Filling factors}

All models are computed in spherical geometry. This means that we assume an atmosphere with a filling factor of hot material of 100 percent. For the Sun this assumption is under debate since the discovery of cool CO in chromospheric layers which lead to thermally bifurcated atmosphere models (e.g. Ayres \& Rabin 1996). For $M$ dwarfs measured magnetic filling factors are often very high: e.g. Saar \& Linsky (1985) measured that about 73 percent of the surface of AD Leo is covered with active regions. For two other very active M4.5 stars JohnsKrull \& Valenti (1996) found magnetic filling factors of $50 \pm$ 13 percent. We tried to infer chromospheric filling factors a posteriori (see Sect. 6.4).

\subsection{Lines used for fitting}

To determine the quality of the fits different lines were chosen for the hotter and brighter stars AD Leo, YZ CMi and $\mathrm{CN}$ Leo as compared to the cool and very faint stars DX Cnc and LHS 3003. For the three mid-type M dwarfs we used the Balmer line series higher than $\mathrm{H}_{9}$, the $\mathrm{NaI} \mathrm{D}$ lines and a number of Fe I and MgI lines between 3650 and $3870 \AA$.
An additional pronounced chromospheric feature is the He I line at $5875 \AA$, which could not be matched for any of the three stars without creating too much flux in the Balmer lines. We ascribe this to our relatively simple approach with nearly linear temperature distributions in the chromosphere and a linear temperature distribution in the TR. This problem applies not only to the He line, but to other lines as well: it is hard to match large parts of the spectrum well with the same model. Therefore our best fit models match most of the lines in principle but not perfectly.

For the two late-type $\mathrm{M}$ dwarfs we used the $\mathrm{H}_{\alpha}$ and the $\mathrm{H}_{\beta}$ line, the Na I D lines and the He I line at $5875 \AA$ since the blue part of the spectra were underexposed for the two stars.

\section{The influence of the model parameters}

Before studying the five $\mathrm{M}$ dwarfs and their best fit model in detail a few words about the influence of the general model parameters and assumptions are in order.

\subsection{Photospheric parameters}

The influence of the underlying photospheric model and its parameters $T_{\text {eff }}$ and $\log g$ has been studied already by Short \& Doyle (1998). They find that lowering or raising $T_{\text {eff }}$ by $200 \mathrm{~K}$ or $\log g$ by 0.5 dex leads to uncertainties in the chromospheric parameters of the colum mass at the onset of the TR or at the temperature minimum of about 0.3 dex. Therefore we used individual photospheric models for each star. Nevertheless we confirm the trend they found comparing the models of AD Leo and YZ CMi (see Sect. 5.2).

Regarding the quality of our photospheric models we estimate the error in $T_{\text {eff }}$ to be about $100 \mathrm{~K}$, since the variation of this parameter led to signifacnt changes in $\chi^{2}$. A comparison of our $T_{\text {eff }}$ values to those published in literature does indeed show agreement to within $100 \mathrm{~K}$ (see Table 1). We also estimate the error in $\log \mathrm{g}$ to be about $0.5 \mathrm{dex}$.

The influence of the stellar mass of the underlying model has not yet been studied to our knowledge. We computed models with 0.5 and $0.1 M_{\odot}$ for our lowest mass star LHS 3003 and found only very weak dependence on this parameter (see Sect. 5.5). Also the influence of metalicity on the chromospheric emission has not been studied so far. Figure 7 strongly suggests, that metalicity should be included in future modelling if it uses metal lines as diagnostics.

\subsection{Chromospheric parameters}

The microturbulence has been shown by Jevremović et al. (2000) to influence the line intensity as well as line shape. The influence of NLTE computations has been found in this article to have not only an influence on emergent flux of the considered species itself, but on other species as well (see Sect. 6.1).

The partial frequency redistribution approximation has not been implemented in PHOENIX so far. Falchi \& Mauas (1998) studied the impact of partial compared to complete frequency redistribution (PRD/CRD) on the $\mathrm{Ly}_{\alpha}$ line. They found that the PRD treatment of $\mathrm{Ly}_{\alpha}$ also influences the Balmer lines. 


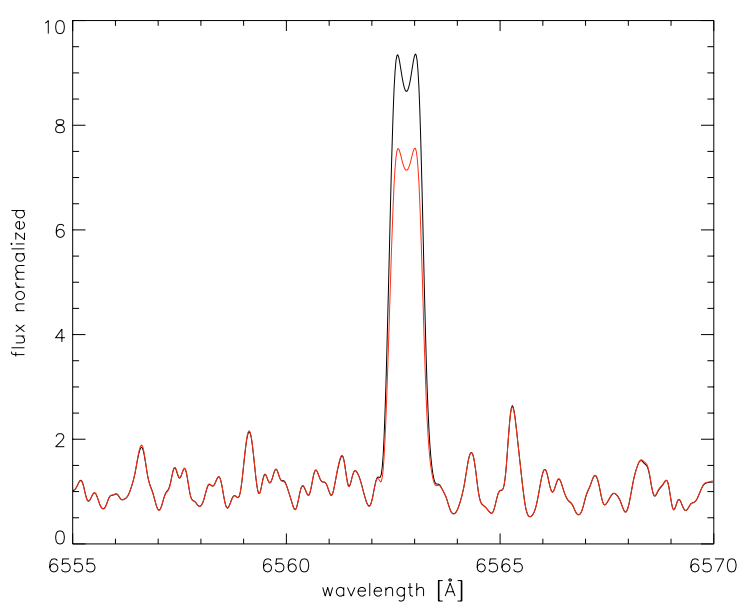

Fig. 3. Comparison of two models with dust (black) and without dust (red). The continuum is normalized for both models, otherwise the difference in the $\mathrm{H}_{\alpha}$ line would cease.

The CRD approximation leads to more emergent flux, therefore our models should have the onset of the TR at too low a pressure compared to a model computed in PRD.

For the cool models with $T_{\text {eff }}=2700 \mathrm{~K}$ and $2500 \mathrm{~K}$ dust may play an important role since it warms the atmosphere in photospheric layers and is an efficient scatterer. For the photospheric spectra themselves the influence of dust is not significant. For the chromospheres we tried two different dust treatments usually used within PHOENIX. In the first approach the dust is treated only in the equation of state, in the second approach the dust is also included in the opacity calculations. While the first approach does not affect the emission lines, the second approach enhances most of the emission lines and lowers the continuum. A comparison can be seen in Fig. 3. Although dust has an impact on the chromosphere modelling we did not use it in the present work since it slows down the computation significantly.

As a last point the use of a linear temperature rise vs. log column mass in the chromosphere should be discussed. Alternatively one could use a non-linear temperature rise as was done by Mauas (2000) or Vernazza et al. (1981) for the Sun using several diagnostics to model different parts of the temperature rise. If there are enough diagnostic lines that correspond directly to the temperature at a certain column mass this is undoubtedly more sensitive to the temperature structure and should give better agreement between data and models. Since NLTE effects play an important role and therefore certain lines can be affected by the radiation of layers far away from their formation depth this is also a more complicated way to build chromospheres. Therefore we refrained from a non-linear temperature rise at the moment.

\section{Results}

The temperature distribution for the best fit models is shown in Fig. 4 for all of the stars and an approximate line formation depth is given for various lines for the model of $\mathrm{AD}$ Leo in Fig. 5. The pronounced emission line of He at $5875 \AA$ is not indicated since the model of AD Leo does not show this line.

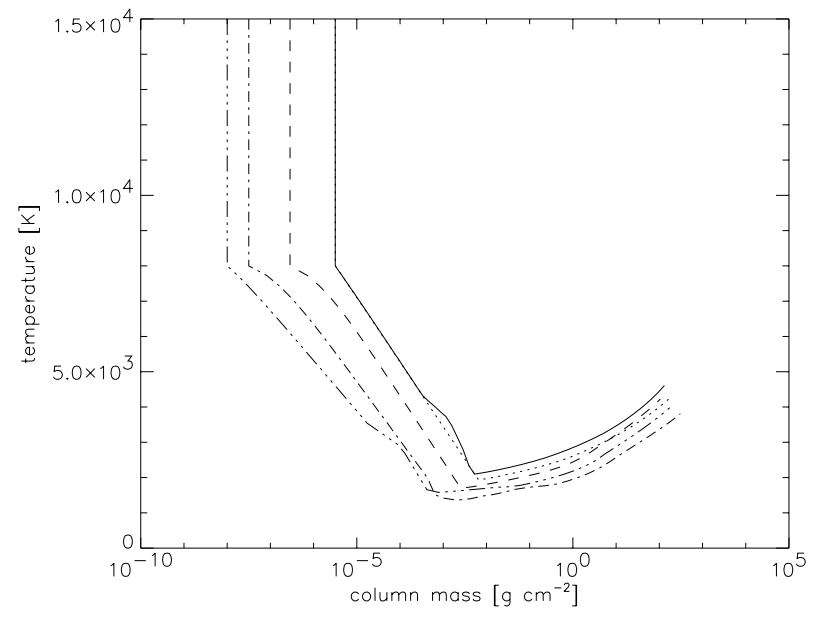

Fig. 4. Temperature structure for our best fit models. The solid line corresponds to $\mathrm{AD} \mathrm{Leo,} \mathrm{the} \mathrm{dotted} \mathrm{line} \mathrm{to} \mathrm{YZ} \mathrm{CMi,} \mathrm{the} \mathrm{dashed} \mathrm{line} \mathrm{to}$ CN Leo, the dot-dashed line to LHS 3003 and the triple-dot-dashed line to DX Cnc. The models for AD Leo and YZ CMi are nearly identical.

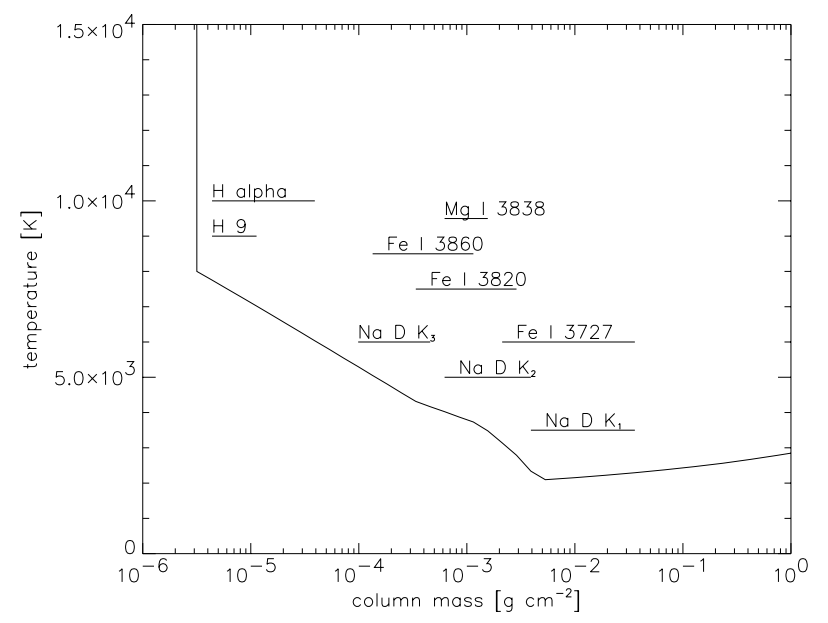

Fig. 5. Temperature structure for our best fit models of AD Leo. Given are the approximate line formation depths for various lines used in the fitting process.

For the determination of the line formation depth we used spectra for each layer in the atmospheric model. The formation of the resulting spectrum in the outermost layer can be observed from layer to layer. One problem with this ansatz is that the net flux is tracked, i.e. the flux going inward is accounted for as well. Therefore the outer boundary of the line formation region can be determined quite well whereas the inner boundary is less certain.

Since for all our observed spectra of the mid-type M dwarfs the $\mathrm{Na}$ I D lines are found as absorption lines with emission cores, the minimum seen in the line profiles can serve as a diagnostic for the location of the temperature minimum in the star's atmosphere (as proposed by Andretta et al. 1997; and Mauas 2000). We also find like Andretta et al. that the Na I D line profile is insensitive to the TR gradient. For the three earlytype $\mathrm{M}$ dwarfs the models show pronounced self absorption in the emission core, which is not seen in the spectra, otherwise the $\mathrm{Na}$ I $\mathrm{D}$ lines are reproduced reasonably well for these stars 
(see e.g. Fig. 9 for YZ CMi). For the two late-type M dwarfs the $\mathrm{Na}$ I $\mathrm{D}$ lines are less well suited to diagnose the temperature minimum since the absorption profile is quite shallow; this is especially true for LHS 3003.

The best fit was determined in two ways, by eye and with a $\chi^{2}$ test using a number of wavelength ranges including the diagnostic lines. For LHS 3003 and DX Cnc the used wavelength ranges are 4855 to 4865,6550 to 6570,5873 to 5877 and 5888 to $5900 \AA$. For AD Leo, YZ CMi and CN Leo the $\mathrm{H}_{\alpha}$ region was omitted and, instead, some of the blue wavelength were used: 3705 to 3728,3825 to 3840,3780 to 3810 and 3850 to 3865 . In the case of AD Leo and YZ CMi both the eye fit as well the $\chi^{2}$ fit resulted in the same best fit model. For CN Leo the $\chi^{2}$ test best fit model differed by $0.1 \mathrm{dex}$ in the TR gradient to the one found by eye, i.e. they are neighbouring models. Also for DX Cnc and LHS 3003 the $\chi^{2}$ test preferred neighbouring models to the by eye fit. We decided to use the models found by eye since for LHS 3003 the $\chi^{2}$ test was contaminated by the $\mathrm{Na} \mathrm{D}$ airglow lines and for DX Cnc we preferred to describe the $\mathrm{H}_{\alpha}$ line more correctly than the $\mathrm{H}_{\beta}$ line (which is the main difference between the two models).

We stopped improving the models when the variation of the three main parameters (column mass at $T_{\min }$, column mass at onset of TR and grad TR) around some starting parameters did not improve the fit. However, no true grid in the parameter space was calculated, since we normally adjusted first the column mass at $T_{\min }$ via the Na D lines, then varied the column mass at the onset of the TR, readjusted the column mass at $T_{\min }$ and then varied the gradient of the TR.

\section{1. $A D$ Leo}

A comparison between model and data for the blue part of the spectrum of AD Leo is shown in Figs. 6 and 7. The complex pattern of pure emission lines and absorption lines with emission cores is reproduced quite well, although the amplitude of most of the lines is not perfectly modelled. The base of the Balmer lines is somewhat broader than in the model but in general the Balmer lines fit reasonably well in amplitude and highest Balmer line seen. Iron lines can be either too strong or too faint, but normally lines from the same multiplet behave in the same way. For example, the three Fe I lines at 3820, 3826 and $3834 \AA$ are all three from the same multiplet and too strong, while the Fe I lines at 3705.5, 3720 and $3722.5 \AA$ from another multiplet are all too faint. Since the deviation of the lines do not vary randomly it is unlikely that it is caused by a lack of reliable atomic data; but even so one should keep in mind that especially the collision rates are usually not well known. The behaviour may be caused by our simple temperature structure. While the $3720 \AA$ line originates in layers corresponding to temperatures between 4500 and $3500 \mathrm{~K}$, the $3820 \AA$ line originates in layers corresponding to temperatures between 3800 and $2800 \mathrm{~K}$. The formation depths are overlapping with the $3820 \AA$ line forming in deeper layers. An alteration of the temperature structure in these layers should improve the fit of these lines. Nevertheless NLTE effects also play a role for these lines, since their amplitudes reacts to the gradient in the TR as well.

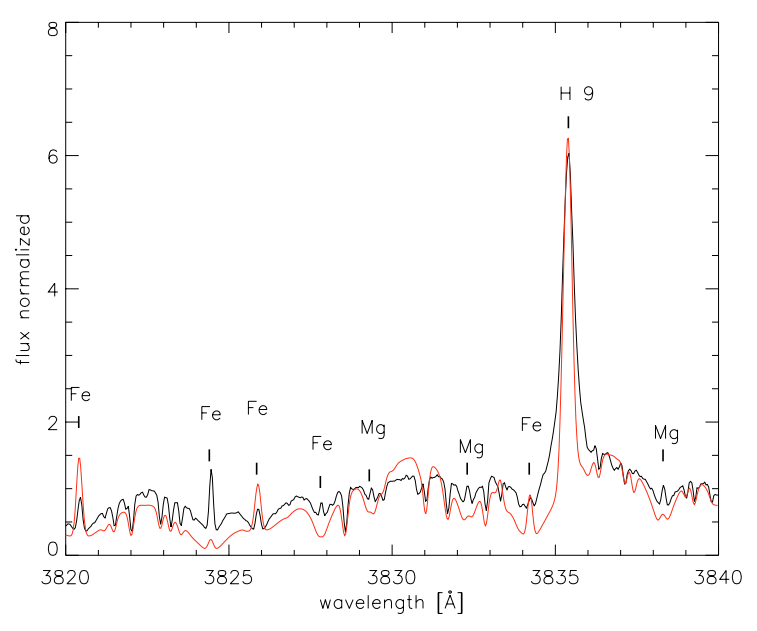

Fig. 6. Comparison of the observed spectrum of AD Leo (black) and the best fit model (grey/red). The emission cores/lines used for the modelling are indicated in the spectrum.

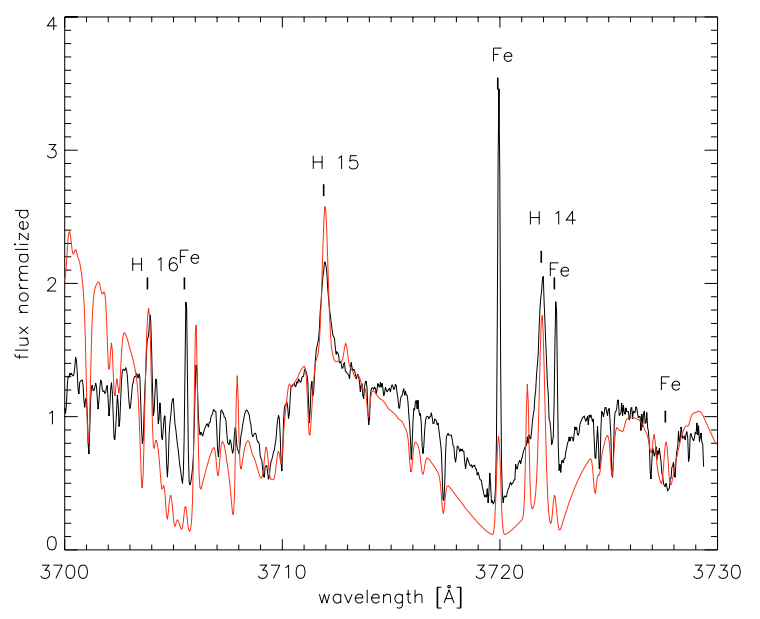

Fig. 7. Same as is in Fig. 6. The absorption part of the Fe I line at $3720 \AA$, is too pronounced in the model what may be due to metalicity.

\section{2. $Y Z C M i$}

For YZ CMi a comparison between model and data can be found in Figs. 8 and 9. The Balmer lines are well fitted in amplitude. Also described correctly is the highest Balmer line that is clearly seen in emission. The base width of the Balmer lines is too narrow in the model. This is worse than for AD Leo and comparable to CN Leo. Since YZ CMi is relatively stable through the observations, pronounced activity is no obvious explanation for this. Since all three stars are very active this discrepancy may be caused by spiculae-like inflows and outflows (see also Sect. 5.5).

As AD Leo, YZ CMi shows a pattern of absorption lines with emission cores which is in general reproduced well. The two stars are very similar, which is reflected in the similar models that differ only by $0.1 \mathrm{dex}$ in the column mass at the temperature minimum and by $0.2 \mathrm{dex}$ in the gradient of the TR. Judging from the spectra (observed or modelled), YZ CMi is the more active star since it shows more emission lines with larger equivalent width. Nevertheless, AD Leo is described by the more active model judged by the temperature minimum 


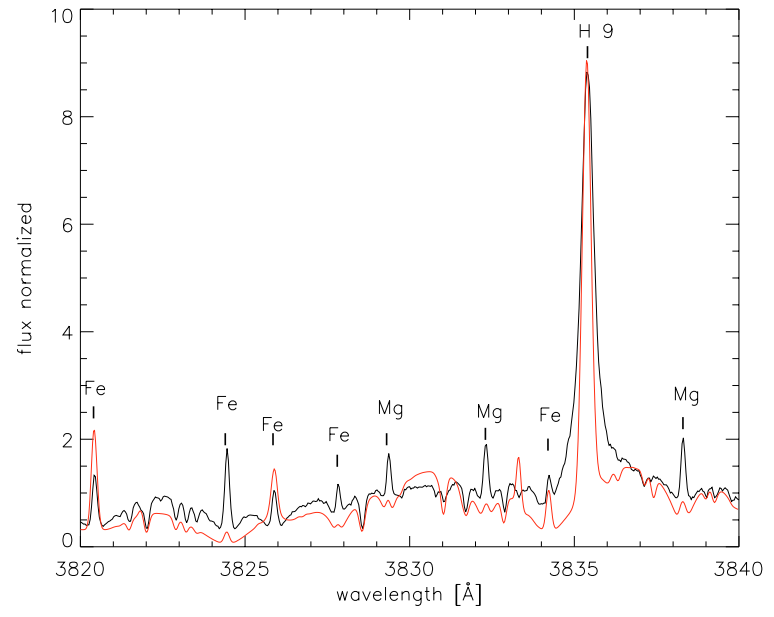

Fig. 8. Comparison of the observed spectrum of YZ CMi (black) and the best fit model (grey/red). The $\mathrm{H}_{9}$ is reproduced quite well. The Mg I lines show all less pronounced emission features in the model compared to the data.

located at higher column mass and the lower gradient in the TR. However in combination with the higher effective temperature of the photospheric model, it actually gives lower amplitudes in the emission lines. Even a temperature difference of $200 \mathrm{~K}$ in the effective temperature of the photosphere can significantly influence the emergent chromospheric flux. Hence a good knowledge of the parameters of the underlying photosphere is essential for chromospheric modelling.

Although the wings of the Na I D lines are fitted reasonably well, the region between the doublet lines is not. This region is sensitive to the temperature minimum and the temperature structure in the low chromosphere, but it is also sensitive to changes in the microturbulent velocity. Also there are deep self-absorption cores in the Na I D lines seen in the models. This may be caused by a wrong temperature structure in the mid chromosphere, where the flux in the line center of the Na I D lines arises (see Fig. 5), but the depth of the selfabsorption core in the $\mathrm{Na}$ I $\mathrm{D}$ line is also strongly dependent on the NLTE set chosen. It may therefore be a pure NLTE effect. In this case there is no strong connection between the temperature structure of a certain part of the model and the strength of the self-absorption.

\subsection{CN Leo}

$\mathrm{CN}$ Leo is the most active star in the sample and has accordingly the worst fit for the individual lines. Nevertheless the disappearance of the absorption lines in favour of pure emission lines is reproduced. While the only $100 \mathrm{~K}$ hotter photosphere of YZ CMi produces many absorption lines with emission cores, the spectrum of CN Leo exhibits pure emission lines - which is fully reproduced by the model.

While the amplitudes of the Balmer lines and the highest Balmer line seen are fitted quite well by the model, the observed Balmer lines are much broader at the baseline than the ones in the model (see Figs. 10 and 11). Although the spectrum used for the modelling is obtained during quiescence there

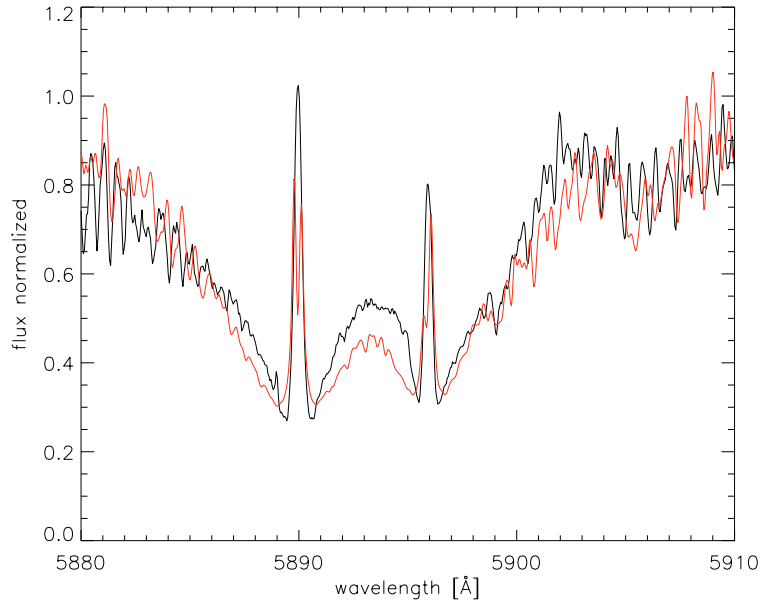

Fig. 9. Same as is in Fig. 8 but for the Na I D lines. While the model shows deep self-absorption this is not seen in the data.

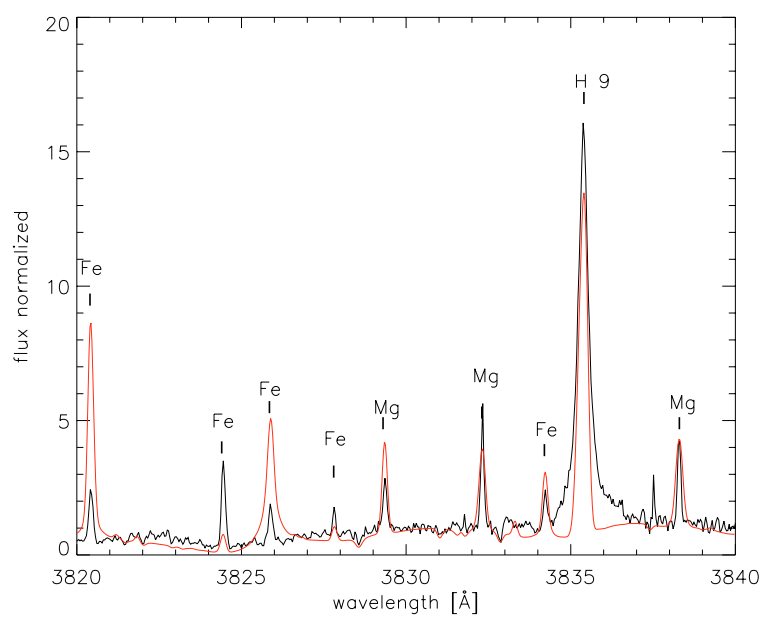

Fig. 10. Comparison between data (black) and model (grey/red) for $\mathrm{CN}$ Leo. Most of the Fe lines are too strong in the model.

may be some activity present providing an additional broadening mechanism. Moreover most of the Fe lines in the model are too strong compared to the data if the Balmer lines are fitted well. On the other hand, models with well fitted iron lines yield too much Balmer line flux. Figure 5 shows that the Fe lines are forming in the middle and lower chromosphere, while the Balmer lines originate from the top of the chromosphere. Therefore a non-linear temperature rise could help to solve this discrepancy. Again the model does not predict He emission lines. This incorrect modelling of the $\mathrm{He}_{3}$ line may be caused by the non-inclusion of a corona in our models as suggested by the work of Mauas et al. (2005).

\subsection{Cnc}

While the He I line at $5875 \AA$ emission line is not predicted by the models for the three mid-type $M$ dwarfs, for the two late-type $\mathrm{M}$ dwarfs the line is too strong in the models. The emission cores of the $\mathrm{NaI} \mathrm{D}$ doublet are far too faint for this star using the linear temperature rise that fit the Balmer lines well (see Fig. 12). Therefore we modified the lower 


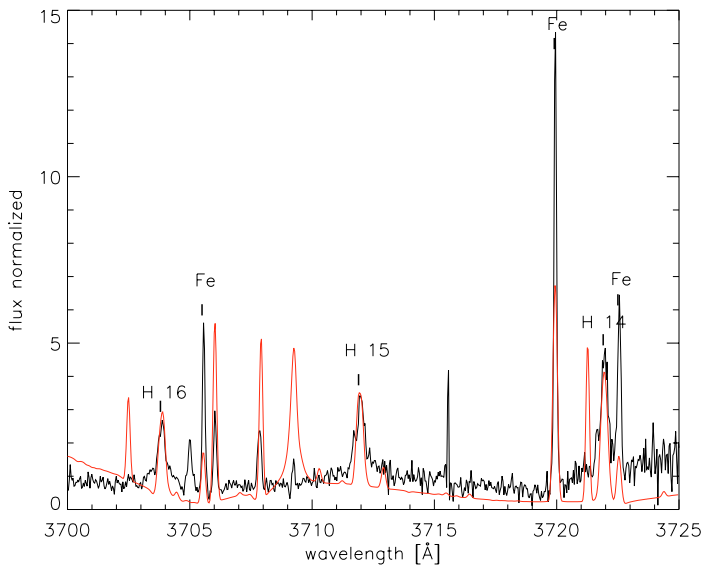

Fig. 11. Same as is in Fig. 10. Again the Fe lines are too strong while the three Balmer lines $\mathrm{H}_{14}$ to $\mathrm{H}_{16}$ are fitted quite well.

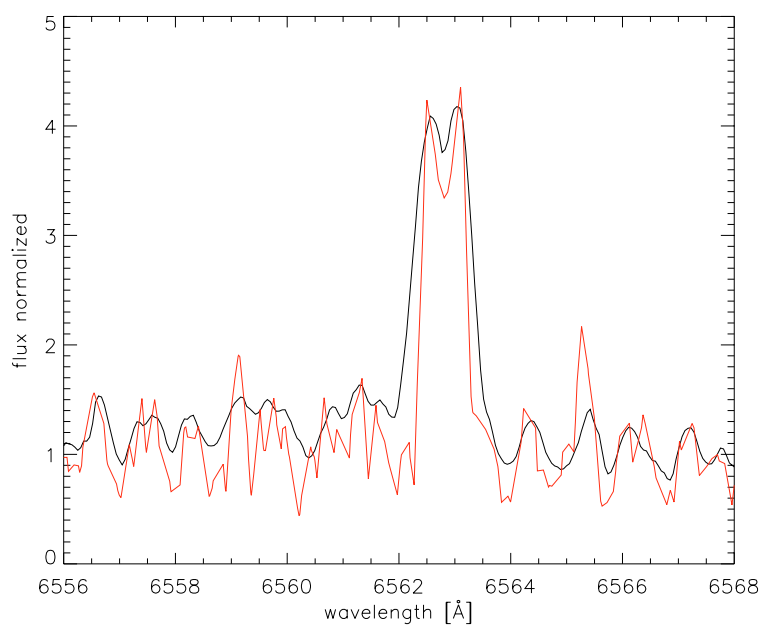

Fig. 12. Comparison of data of DX Cnc (black) and best fit model (grey/red) for the $\mathrm{H}_{\alpha}$ line. The model shows too deep self-absorption.

chromosphere temperature structure and started with a higher gradient directly at the temperature minimum, but joined the original temperature rise at about $4000 \mathrm{~K}$ before hydrogen starts to ionize. This leaves the Balmer lines nearly unaltered and gives stronger emission cores in the $\mathrm{Na} \mathrm{D}$ doublet.

\subsection{LHS 3003}

While all other stars were computed with $0.5 M_{\odot}$, LHS 3003 was computed with $0.1 M_{\odot}$ because of its low effective temperature and surface gravity and to test the influence of the mass on the chromospheric model. While a higher mass produces a higher Ly $\alpha$ line, the Balmer series lines are slightly lower. Therefore we conclude that the star's mass in the model has an influence on the chromospheric model but the effect is small compared to other parameters and NLTE effects.

While the amplitude of the $\mathrm{Na}$ I D lines is well fitted, the lines are too wide at the line base in the model (see Fig. 14). The width of the line is also not fitted well for the $\mathrm{H}_{\alpha}$, and for the $\mathrm{H}_{\beta}$ line: these two lines are too narrow in the model as can be seen in Fig. 13 for the $\mathrm{H}_{\alpha}$ line. This may be due to the rotational velocity of the star. To test this hypothesis

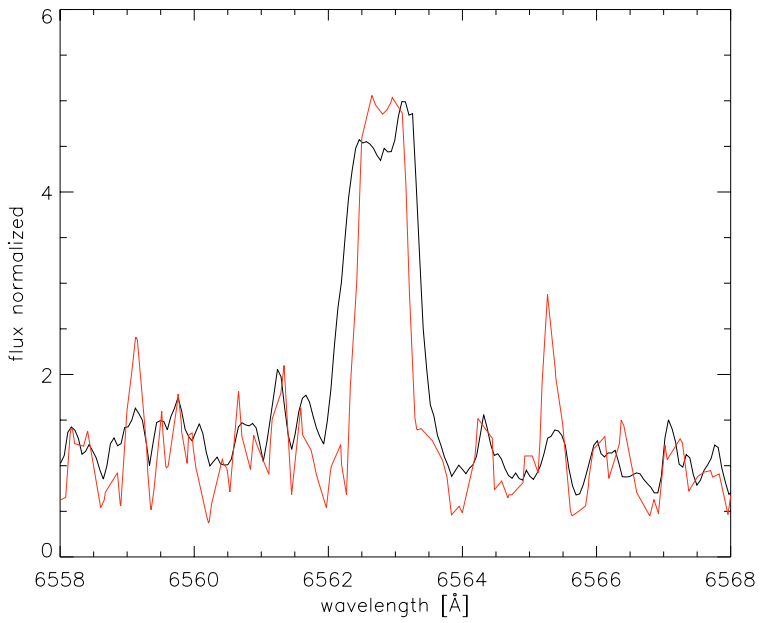

Fig. 13. Comparison of data of LHS 3003 (black) and the best fit model (grey/red) for the $\mathrm{H}_{\alpha}$ line. Here the self-absorption in the model is not deep enough.

we spun up the model to $20-30 \mathrm{~km} \mathrm{~s}^{-1}$ to fit the line width of the $\mathrm{H}_{\alpha}$ line. On the other hand, we measured the rotational velocity of LHS 3003 in the photospheric lines using CN Leo as a template (see Fuhrmeister et al. 2004, for the method used); this procedure leads to a rotational velocity of $6.0 \pm 1.5 \mathrm{~km} \mathrm{~s}^{-1}$. If one compares the photospheric features in Fig. 13 next to the $\mathrm{H}_{\alpha}$ line to the model (with no rotation at all), a slow rotational velocity seems to be very reasonable. Moreover this additional broadening is not seen in other emission lines e.g. the Na I D lines. Therefore rotational broadening can be ruled out and the chromospheric emission line must be affected by another broadening mechanism. The modelling includes Stark and van der Waals broadening approximations. The self-broadening of the Balmer lines may, however, not be described correctly (Barklem et al. 2000). Another possibility ascribes the additional broadening to a more dynamic scenario with mass motions. If the star hosts several active regions exhibiting mass motions, the overlapping of the lines would lead to an additional broadening. Also spiculae-like inflows and outflows may contribute to Balmer line broadening as described for II Peg by Short et al. (1998). A dynamic scenario would also explain the asymmetric profile of both the $\mathrm{H}_{\alpha}$ and the $\mathrm{H}_{\beta}$ line in the star, which is not reproduced by any of our models. Since the $\mathrm{H}_{\alpha}$ line shows two prominent components we tried to fit the line with two Gaussians leading to Doppler shifts of $-11 \mathrm{~km} \mathrm{~s}^{-1}$ and $16 \mathrm{~km} \mathrm{~s}^{-1}$, respectively, where we used $6562.81 \AA$ as reference central wavelength. The $\mathrm{H}_{\beta}$ line is not composed of two components, but fitting it with three Gaussian components leads to one component at about rest wavelength and two components with Doppler shifts of about $-11 \mathrm{~km} \mathrm{~s}^{-1}$ and $18 \mathrm{~km} \mathrm{~s}^{-1}$ using $4861.33 \AA$ as rest wavelength. Moreover the three averaged spectra show some changes during the time series in the line profiles of the two Balmer lines. Since the photometer shows no major flaring activity it seems that the quiescent activity of LHS 3003 is composed of different active regions. 


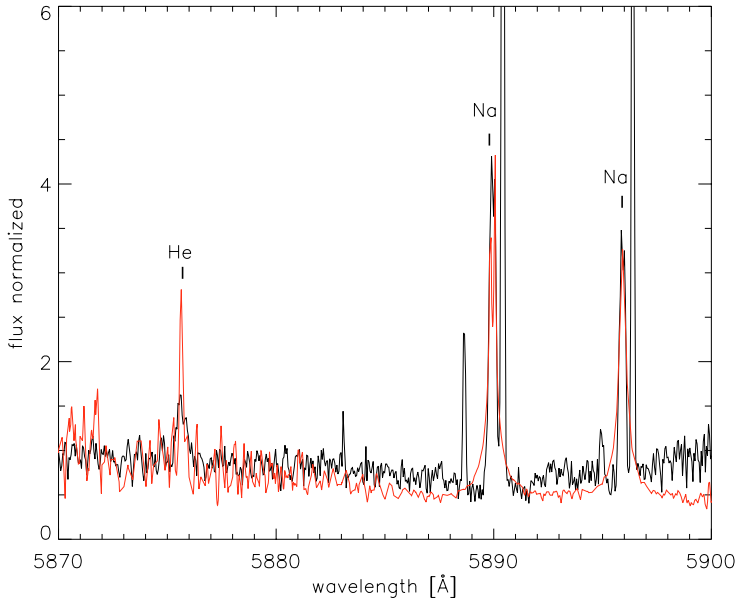

Fig. 14. Same as is in Fig. 13 but for the Na I D lines and the He I line at $5875 \AA$. The narrow emission lines are known airglow lines that have not been removed (see also Fig. A.1).

\section{Discussion}

\subsection{NLTE effects}

The line flux and the line profile are both sensitive to NLTE effects. Therefore it is necessary to compute at least all species in NLTE whose lines are used for the model fitting. Lines computed in LTE usually show too strong emission as in Fig. 15. However, the lines are not only influenced by the NLTE calculations of the line forming species itself but by other species as well. For example, the Balmer lines and the $\mathrm{Na}$ I $\mathrm{D}$ lines are influenced by the NLTE computations of carbon, nitrogen and oxygen (CNO). Therefore as many species as possible should be treated in NLTE. To investigate the influence of different species on each other, we computed for YZ CMi model atmospheres and spectra with different NLTE sets. Hydrogen, helium and $\mathrm{Na}$ I to Na IV were always computed in NLTE. In addition we computed the same model with CNO I to CNO III in NLTE, and with CI to VI, N I to N V, O I to VI in NLTE. The latter NLTE set was chosen to cover all ions of CNO with significant partial pressures in the temperature domain below $\log T=5.0$. Moreover we computed the same model with different less abundant metals in NLTE (in addition to CNO I to CNO III): one set chosen was Fe I to Fe VII, Mg I to Mg IV and Ni I to Ni III, the other one Fe I to Fe IV and Co I to Co III. The latter two models did not differ significantly in the Balmer lines nor in the Fe I lines. The difference in the $\mathrm{NaI} \mathrm{D}$ lines is less than about 10 percent. The electron density for these two models are very similar except in a region around the temperature minimum, which is seen in the variation of the $\mathrm{Na}$ I D lines in the spectrum. The comparison of one of these two models with CNO I to CNO III in NLTE shows much larger differences both in the electron density and in the spectrum. Therefore we conclude, that it is important to treat at least Fe I to Fe IV in NLTE. The largest changes can be seen in the amplitudes of the higher Balmer lines and in the $\mathrm{Na}$ I $\mathrm{D}$ lines which is about 30 percent while for the $\mathrm{H}_{\alpha}$ and $\mathrm{H}_{\beta}$ line the difference is about 10 percent.

Even more important is the influence of the treatment of the CNO ions. Between no CNO NLTE treatment at all and the first

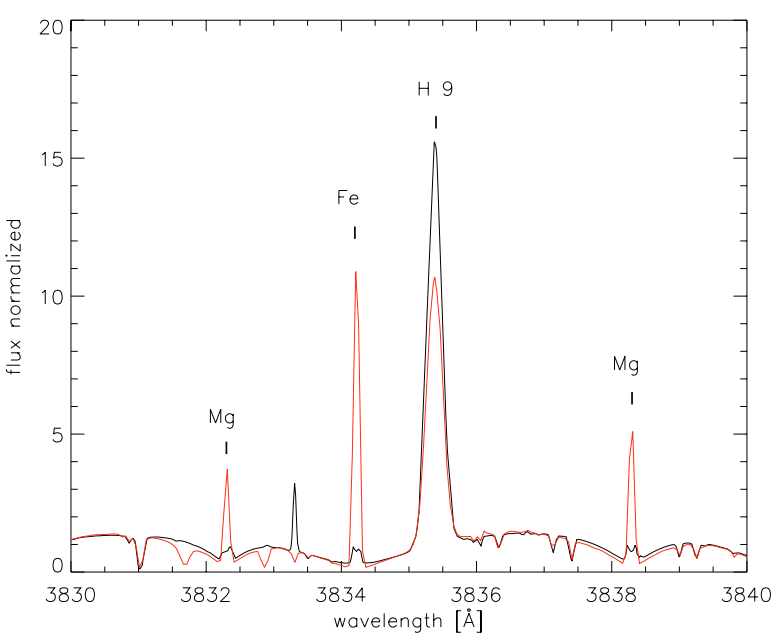

Fig. 15. Comparison of two computations with different NLTE sets. The line at $3835.4 \AA$ is $\mathrm{H}_{9}$, the other lines are Fe I and Mg I. The Fe I and $\mathrm{Mg}$ I lines in the grey/red model often show much stronger line flux than in the black model since they are not computed in NLTE in the grey/red model. The $\mathrm{H}_{9}$ line is influenced by the different NLTE sets of the two models, though hydrogen is computed in NLTE for both models. Further computations show that this is caused mainly by the different treatment of $\mathrm{CNO}$ in the two models.

two ions in NLTE the amplitudes of the Balmer lines change dramatically. While for the $\mathrm{H}_{9}$ line the amplitude is about doubled, the amplitude of the $\mathrm{H}_{\beta}$ line is about halved, the amplitude of the $\mathrm{Na}$ I D lines is also about halved. For further CNO ions treated in NLTE the flux in the $\mathrm{H}_{\alpha}$ and $\mathrm{H}_{\beta}$ line decays further, while the amplitude of the $\mathrm{Na}$ I D lines and the higher Balmer lines stays nearly constant. The treatment of the higher $\mathrm{CNO}$ ions in NLTE is very problematic since these ions are in principle not present in most parts of the atmosphere and therefore the code must deal with very tiny numbers.

The compensation for the additional flux in the models with the large NLTE sets were usually done via adapting the gradient in the TR. Typically this gradient had to be increased for about 0.2 dex to compensate for the additional emergent flux in the Balmer series.

One explanation of this effect is through the direct influence of the NLTE calculations on the electron density: NLTE calculations of one species influence the electron density and therefore the level population of all other species. Another possibility is the influence of a species with a multitude of lines in the hydrogen continuum region which would influence the hydrogen ionisation equilibrium and therefore indirectly the electron density. Since cobalt and nickel have many lines in the hydrogen continuum region and these species do not influence the electron density too much, this seems to be a second order effect compared to the direct influence of the electron density via the ionisation balance of the more abundant elements (see Fig. 17). On the other hand, the NLTE computation of the Fe ion levels influence the electron density in large parts of the atmosphere.

To gain some further insight in the NLTE behaviour of chromospheres we built a simple chromosphere model for the Sun that is supposed to be closer to LTE than M dwarfs. 


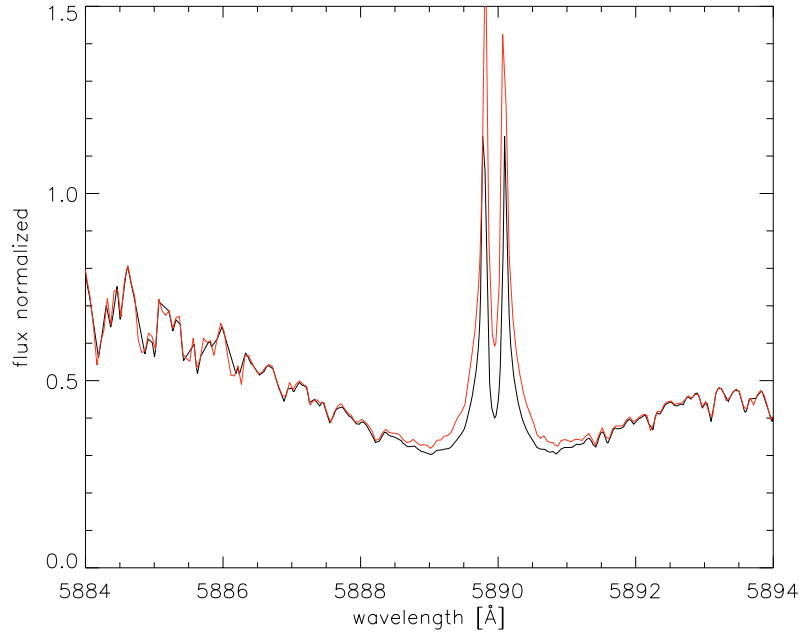

Fig. 16. Same comparison as in Fig. 15 for the Na I D line. The NLTE set that also includes $\mathrm{Fe}, \mathrm{Mg}$ and $\mathrm{CNO}$ show shallower $\mathrm{Na}$ emission (black line) than the one that treats only $\mathrm{H}, \mathrm{He}$ and Na in NLTE (grey/red line).

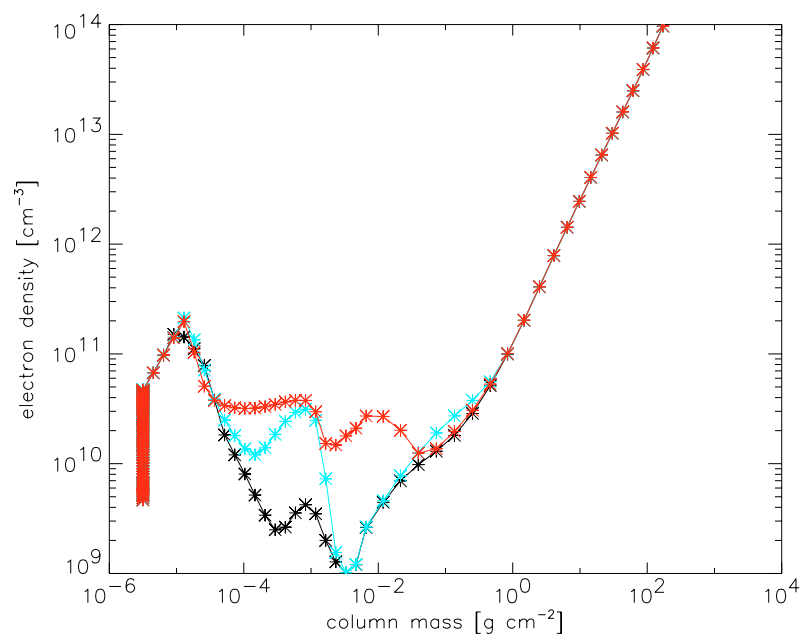

Fig. 17. Comparison of the influence of different NLTE sets on the electron density. The black line is the basic model with $\mathrm{H}, \mathrm{He}$ and $\mathrm{Na}$ in NLTE. Grey/red is the model with $\mathrm{Fe} / \mathrm{Ni}$ in NLTE and light grey/turquoise is the model with CNO I to CNO III in NLTE. All three models differ significantly in large parts of the atmosphere.

We computed a model with $\mathrm{H}, \mathrm{He}$ and $\mathrm{Ca} \mathrm{I}-\mathrm{V}$ and a model with $\mathrm{H}, \mathrm{He}, \mathrm{Ca}$ I-III, CNO I-III, and Fe I-III in NLTE. The two models show very little differences in the Balmer lines and in the $\mathrm{Ca}$ II $\mathrm{H}$ and $\mathrm{K}$ line. The largest variation seen in these lines is about 10 percent which is indeed much less than in the $\mathrm{M}$ dwarfs. Therefore the strong variations due to the set of NLTE species chosen is characteristic of the M dwarf models. Although this does not rule out computational artefacts it strengthens our confidence in the reliability of the variations in the electron pressure and the spectral lines in our M dwarfs.

\subsection{Uniqueness of the models}

During our chromospheric modelling we encountered the problem of non-uniqueness of the models in several ways, but it could be resolved each time. Since the $\mathrm{Na} \mathrm{D}$ lines are known to be only sensitive to changes in the lower chromosphere, clearly an additional diagnostic is needed. The Balmer lines are sensitive to changes in the upper chromosphere and the gradient in the TR. Nevertheless we found models with nearly identical Balmer lines either in the lines higher than $\mathrm{H}_{9}$ or in $\mathrm{H}_{\beta}$ and $\mathrm{H}_{\alpha}$ but normally not both. Therefore the very high Balmer lines seems to be an important diagnostic tool. Only for different NLTE sets the whole Balmer series can be nearly identical for different model parameters. Since e.g. the Fe lines are not reproduced correctly by a LTE treatment, the models can be distinguished by these lines.

Since we used only $\mathrm{H}_{\alpha}, \mathrm{H}_{\beta}$ and the $\mathrm{Na} \mathrm{D}$ lines for the model construction of LHS 3003 and DX Cnc, these models may be affected by non-uniqueness. However since the emission lines react more sensitively to all chromospheric parameters when lowering $T_{\text {eff }}$ we do not regard this as a serious problem.

Another interesting point is to test if the photospheric parameters used may introduce some kind of non-uniqueness. Since AD Leo and YZ CMi have the most similar photospheric spectra in the sample, we tested if the spectrum of YZ CMi could be fitted with one of the model spectra created for AD Leo that has a hotter underlying photosphere than YZ CMi and the same $\log g$. We found that the models with the hotter photosphere give poorer fits since a stronger chromosphere was needed to match the Balmer lines, which led to too much flux in the metal lines. Since the chromospheric parameter space is designed for AD Leo, though, we may miss a well fitting model with a hotter photosphere for YZ CMi.

\subsection{Comparison to other models}

Our hottest sample star AD Leo is the only one in our sample for which chromosphere models have been computed by other authors. Mauas \& Falchi (1994) derived a semi-empirical model of the photosphere and chromosphere of the quiescent state of AD Leo fitting the continuum as well as some chromospheric lines. Hawley \& Fisher (1992) constructed a grid of flare models for AD Leo including a quiescent one. These models were constructed on a photospheric base by using X-ray heating from a model for the overlying corona. Short \& Doyle (1998) constructed chromospheric and TR models in a very similar way as our approach, using a linear temperature vs. $\log m$ distribution. Moreover, all these authors used very different underlying photospheres. While Mauas \& Falchi (1994) fitted both photosphere and chromosphere at the same time semiempirically and no $T_{\text {eff }}$ or $\log g$ values were given, Hawley \& Fisher used a model by Mould (1976) with $T_{\text {eff }}=3500 \mathrm{~K}$ and $\log g=4.75$, and Short \& Doyle used a PHOENIX photosphere with $T_{\text {eff }}=3700 \mathrm{~K}$ and $\log g=4.7$. All authors used solar chemical composition as we did. Since the emission lines are sensitive to the underlying photospheres as mentioned above and discussed in Short \& Doyle (1998), and due to the different construction all these models are difficult to compare. The models of Short \& Doyle (1998) are very similar to our own models, although they used a photosphere considerably hotter. Accordingly they found a TR onset at higher pressure than we did. 
Table 2. Filling factors of the chromospheres for the modelled stars and the parameters used to calculate the filling factors. The measured scaling factor $f_{\mathrm{m}}$ between model and flux calibrated spectrum is given as well as the theoretical scaling factor $f_{\mathrm{th}}=R^{2} / d^{2}$.

\begin{tabular}{ccccccc}
\hline \hline Star & $\log L_{\text {bol }}$ & Radius [cm] & distance [pc] & $f_{\mathrm{m}}$ & $f_{\text {th }}$ & Filling factor \\
\hline AD Leo & $31.94^{1}$ & $3.4 \times 10^{10}$ & $4.9^{1}$ & $3.0 \times 10^{-26}$ & $5.3 \times 10^{-26}$ & 0.57 \\
YZ CMi & $31.69^{1}$ & $2.9 \times 10^{10}$ & $6.1^{1}$ & $1.7 \times 10^{-26}$ & $2.5 \times 10^{-26}$ & 0.68 \\
CN Leo & $30.55^{1}$ & $8.4 \times 10^{9}$ & $2.4^{1}$ & $0.7 \times 10^{-26}$ & $1.4 \times 10^{-26}$ & 0.50 \\
DX Cnc & $30.48^{1}$ & $8.9 \times 10^{9}$ & $3.6^{1}$ & $1.7 \times 10^{-27}$ & $6.6 \times 10^{-27}$ & 0.26 \\
LHS 3003 & $30.31^{2}$ & $8.5 \times 10^{10}$ & $6.4^{3}$ & $0.5 \times 10^{-27}$ & $1.9 \times 10^{-27}$ & 0.26 \\
\hline
\end{tabular}

${ }^{1}$ Delfosse et al. (1998), ${ }^{2}$ Leggett et al. (2002), ${ }^{3}$ Dahn et al. (2002).

\subsection{Filling factors}

Our one dimensional model atmospheres correspond to a 100 percent filling factor of chromospheric material. Nevertheless one can estimate the filling factor a posteriori. With distance and radius of the star known, the theoretical scaling factor between flux at the stars surface and flux at earth atmosphere can be calculated. This can be compared to the scaling factor between the model and the flux calibrated spectrum; the ratio of the two gives the filling factor of the chromosphere. We calculated the radius of the stars from the measured luminosity and the temperature of the photospheric model via $L_{\mathrm{bol}}=4 \pi R^{2} \sigma T_{\mathrm{eff}}$. The largest uncertainty is caused by the flux calibrations since the standard star was observed only once per night. We estimate the error in the absolute fluxes to be as large as a factor of two. The calculated filling factors can be found in Table 2. Due to the possibly big errors in flux calibration the filling factors should be considered only as estimates, nevertheless, the three early and mid-type $M$ dwarfs seem to have higher filling factors than the two late-type dwarfs.

The low chromospheric filling factors of the two late-type M dwarfs imply that one-dimensional model calculations may be a poor approximation, since a significant portion of the energy in the active regions will be radiated away horizontally. Therefore stronger chromospheres than we have inferred here may be embedded in cool material. For proper simulations of such a chromosphere three-dimensional hydrodynamical simulations would be needed, as is done for the Sun, e.g. by Wedemeyer et al. (2004).

\section{Summary and conclusion}

We have presented the first semi-empirical models for mid- and late-type M dwarfs accounting for Fe I lines. We found that the models are able to fit the transition from absorption lines with emission cores to pure emission lines at about 3000/2900 K effective temperature. Moreover we found models for five individual stars with effective temperature between 3200 and $2500 \mathrm{~K}$ that fit many spectral features reasonably well. We found for Fe I lines from the same multiplet that they normally behave the same way: the model predicts too high or too shallow amplitudes for all of them. Therefore some of the multiplets may be used to obtain more complex temperature distributions that are able to fit the spectral lines even better.

Moreover we investigated the behaviour of the emerging spectrum under NLTE calculations of different species and found a large influence especially of $\mathrm{CNO}$, that may alter the Balmer and Na I D lines significantly. There is far less NLTE crosstalk caused by species like $\mathrm{Co}, \mathrm{Ni}$ and $\mathrm{Ti}$.

For the two late-type M dwarfs DX Cnc and LHS 3003 we studied the behaviour of the models if dust is considered and found that dust can affect the emerging emission lines if it is considered not only in the equation of state but also in the opacity calculations.

These very late type objects can be described by the same type of chromospheric model atmosphere as the earlier $\mathbf{M}$ dwarfs except that the onsets of the chromosphere as well as the TR move to lower pressure. Thus, there seem to be no principal differences in the heating mechanisms of the chromospheres down to M7. This is relevant in the context of the ongoing discussion about the decreasing activity of the very late-type objects and in particular whether they have possibly only transient chromospheres and coronae. Since the decrease in activity starts at around M7, even more late-type objects than hitherto investigated should be included in chromospheric modelling attempts.

We therefore conclude that modelling of chromospheres with semi-empirical deduced temperature distributions relies heavily on correct input parameters and model assumptions such as effective temperature of the photosphere, $\log g$, NLTE treatment of important lines and dust treatment for the coolest stars. Another parameter probably as important as the others is the metallicity which is normally not considered in chromospheric modelling.

Acknowledgements. Most of the model computations were performed at the Norddeutscher Verbund für Hoch- und Höchstleistungsrechnen (HLRN) and at the Hamburger Sternwarte Apple G5 cluster financially supported by a HBFG grant.

B. Fuhrmeister acknowledges financial support by the Deutsche Forschungsgemeinschaft under DFG SCHM 1032/16-3, and thanks A. Schweitzer for numerous discussions that contributed to this work. P.H.H. was supported in part by the Pôle Scientifique de Modélisation Numérique at ENS-Lyon.

\section{References}

Andretta, V., Doyle, J. G., \& Byrne, P. B. 1997, A\&A, 322, 266

Avrett, E. H., \& Loeser, R. 1984, in Methods in Radiative Transfer, 341

Ayres, T. R. 1979, ApJ, 228, 509

Ayres, T. R., \& Rabin, D. 1996, ApJ, 460, 1042

Barklem, P. S., Piskunov, N., \& O’Mara, B. J. 2000, A\&A, 363, 1091 
Carlsson, M. 1986, Uppsala Observatory Internal Report No. 33

Carlsson, M., \& Stein, R. F. 2002, ApJ, 572, 626

Cram, L. E., \& Mullan, D. J. 1979, ApJ, 234, 579

Dahn, C. C., Harris, H. C., Vrba, F. J., et al. 2002, AJ, 124, 1170

Delfosse, X., Forveille, T., Perrier, C., \& Mayor, M. 1998, A\&A, 331, 581

Falchi, A., \& Mauas, P. J. D. 1998, A\&A, 336, 281

Fuhrmeister, B., \& Schmitt, J. H. M. M. 2004, A\&A, 420, 1079

Fuhrmeister, B., Schmitt, J. H. M. M., \& Wichmann, R. 2004, A\&A, 417, 701

Gizis, J. E., Monet, D. G., Reid, I. N., et al. 2000, AJ, 120, 1085

Hall, P. B. 2002, ApJ, 580, L77

Hanuschik, R. W. 2003, A\&A, 407, 1157

Hauschildt, P. H., Allard, F., \& Baron, E. 1999, ApJ, 512, 377

Hawley, S. L., \& Fisher, G. H. 1992, ApJS, 78, 565

Jevremović, D., Doyle, J. G., \& Short, C. I. 2000, A\&A, 358, 575

Johns-Krull, C. M., \& Valenti, J. A. 1996, ApJ, 459, L95

Jones, H. R. A., Longmore, A. J., Allard, F., \& Hauschildt, P. H. 1996, MNRAS, 280, 77

Jordan, C., Ayres, T. R., Brown, A., Linsky, J. L., \& Simon, T. 1987, MNRAS, 225, 903
Kurucz, R. L., \& Bell, B. 1995, Atomic line list, Kurucz CD-ROM, Cambridge, MA: Smithsonian Astrophysical Observatory, c1995, April 15

Leggett, S. K., Golimowski, D. A., Fan, X., et al. 2002, ApJ, 564, 452

Liebert, J., Kirkpatrick, J. D., Cruz, K. L., et al. 2003, AJ, 125, 343

Mauas, P. J. D. 2000, ApJ, 539, 858

Mauas, P. J. D., \& Falchi, A. 1994, A\&A, 281, 129

Mauas, P. J. D., Andretta, V., Falchi, A., et al. 2005, ApJ, 619, 604

Mihalas, D. 1978, Stellar atmospheres, 2nd edition (San Francisco, W. H. Freeman and Co.), 650

Mohanty, S., \& Basri, G. 2003, ApJ, 583, 451

Moore, C. E. 1972, Nat. Stand. Ref. Data Ser., 40

Mould, J. R. 1976, A\&A, 48, 443

Saar, S. H., \& Linsky, J. L. 1985, ApJ, 299, L47

Short, C. I., \& Doyle, J. G. 1997, A\&A, 326, 287

Short, C. I., \& Doyle, J. G. 1998, A\&A, 336, 613

Short, C. I., Byrne, P. B., \& Panagi, P. M. 1998, A\&A, 338, 191

Vernazza, J. E., Avrett, E. H., \& Loeser, R. 1981, ApJS, 45, 635

Wedemeyer, S., Freytag, B., Steffen, M., Ludwig, H.-G., \& Holweger, H. 2004, A\&A, 414, 1121

Young, P. R., Del Zanna, G., Landi, E., et al. 2003, ApJS, 144, 135 


\section{Online Material}




\section{Appendix A: Identification of chromospheric emission lines}

Chromospheres of $\mathrm{M}$ dwarfs exhibit hundreds of emission lines especially in the near UV between 3000 and $4000 \AA$. These lines can even be multiplied during flares. Deciding which lines to use for the chromospheric modelling made us produce an extensive emission line list for the near UV and optical. We restricted that list not to the 5 modelled M dwarfs but used the whole sample of 21 late-type stars and brown dwarfs. 527 different emission lines of the elements $\mathrm{H}, \mathrm{He}, \mathrm{Na}, \mathrm{Mg}, \mathrm{Al}, \mathrm{Si}, \mathrm{K}, \mathrm{Ca}, \mathrm{Sc}, \mathrm{Ti}, \mathrm{Cr}, \mathrm{Mn}, \mathrm{Fe}, \mathrm{Co}, \mathrm{Ni}$ could be identified, revealing the different levels of activity in the stars.

The observation parameters can be found in Table A.1. For further information about the observations and the data analysis see the article above or Fuhrmeister et al. (2004).

IRAF was used to measure the central wavelength, FWHM and the equivalent width $(E W)$ of the emission lines. We decided to fit the background by eye since otherwise emission cores could not be treated and there are wavelength ranges where the lines are so crowded that it is hard to find appropriate pseudo-continuum points. Therefore the $E W$ measurements are affected by a rather large error. For single lines in spectral wavelength ranges with an ill defined continuum this error may be as big as a factor of two, but for most lines it is less than 40 percent.

We identified the emission lines with the help of the Moore catalog (Moore 1972). Few lines were identified via the NIST database $^{1}$. Moreover we re-identified a random sample of about 5 percent of the lines with the help of the PHOENIX atmosphere models and found full agreement. Of the identified lines 369 are in the blue arm and 158 are in the red arm of the spectra. In the electronically published Table A. 2 the central wavelength of the lines as well as the FWHM and the $E W$ can be found.

All spectra besides the brown dwarfs were shifted to rest wavelength before the measurements of the lines. Therefore the main problem was the recognition what is an emission line or emission core. This suffers from the low signal to noise ratio especially in the blue end of the spectra where the count rates are very low. In addition in some wavelength ranges the continuum is not well defined and in these regions it is sometimes hard to decide whether a particular feature is an emission line or barely left over continuum between consecutive absorption lines. Since we excluded doubtful features from our line list, the list cannot be claimed to be complete for weaker lines.

We provide some remarks on individual stars:

\section{A.1. GJ $229 A$}

This is the earliest star in the sample and rather inactive. It shows only Fe I and Fe II emission cores and no pure emission lines at all.

\footnotetext{
1 Available online under http://physics.nist.gov/ cgi-bin/AtData/main_asd
}

\section{A.2. AD Leo, YZ CMi, Prox Cen and LHS 292}

The activity level from AD Leo can be compared to YZ CMi, Prox Cen and LHS 292. All three stars exhibit about the same number of Fe I, Fe II and other metal lines, but AD Leo and LHS 292 show very few Ni I emission lines compared to the other two stars. LHS 292 also shows a reduced number of Ti II lines.

\section{A.3. AT Mic, FN Vir and GL Vir}

The reduced number of emission lines in these stars is at least partly due to the much lower $\mathrm{S} / \mathrm{N}$ ratio compared to AD Leo, YZ CMi and Prox Cen.

\section{A.4. UV Cet}

UV Cet is a fast rotator with $v \sin i=32.5 \mathrm{~km} \mathrm{~s}^{-1}$ (Mohanty $\&$ Basri 2003). Superimposed on the broad emission lines is a set of slightly redshifted, narrow emission lines. We tentatively interprete this as emission from a chromospheric active region (Fuhrmeister et al. 2004).

\section{A.5. CN Leo}

Besides the line list for the averaged spectrum of $\mathrm{CN}$ Leo we produced line-lists for a one hour averaged time series on 2002 March, 16th containing a short duration flare and a line list for an one hour averaged time series on 2002 March, 15th showing enhanced emission line flux but no pronounced variablity in the light curve. Since the flare was only a short duration flare this line list is showing actually less emission lines than the averaged one, since CN Leo showed less emission lines directly before and after the flare.

\section{A.6. LHS 2076}

The star exhibited a short duration flare during the observations and for the single flare spectrum an additional line list was created. While the number of the Fe I lines remain nearly unchanged during the flare, the number of Ti II lines increases from 2 to 11 and the number of $\mathrm{Ni}$ I from 4 to 13 lines revealing temperature changes in the chromosphere during the flare. During the flare the lines are statistically redshifted by an amount of about $0.05 \AA$.

\section{A.7. LHS 2034}

The star underwent a long duration flare during the observation. We created an additional line list for the first spectrum taken containing the flare peak. This flare spectrum shows particularly many emission lines in the red part of the spectrum - where the other stars show only very few lines. No line shifts could be found for the flare spectrum. Since the flare decays very slowly the averaged spectrum is affected by the flare spectrum. 
Table A.1. Basic observations parameters of the observed stars.

\begin{tabular}{|c|c|c|c|c|}
\hline Name & $\begin{array}{l}\text { Other } \\
\text { name }\end{array}$ & $\begin{array}{l}\text { Spectral } \\
\text { type }\end{array}$ & Observations & $\begin{array}{l}\text { Number of } \\
\text { identified lines }\end{array}$ \\
\hline LHS 1827 & GJ 229A & M1 & $2002-03-154$ spectra $1200 \mathrm{~s}$ & 11 \\
\hline LHS 5167 & AD Leo & M3.5 & $\begin{array}{l}2002-03-133 \text { spectra } 1800 \mathrm{~s} \\
2002-03-162 \text { spectra } 1200 \mathrm{~s}\end{array}$ & 142 \\
\hline HD 196982 & AT Mic & M 4.5 & 2002-03-16 2 spectra $2400 \mathrm{~s}$ & 91 \\
\hline LHS 1943 & YZ CMi & M4.5e & 2002-03-13 3 spectra $3600 \mathrm{~s}$ & 178 \\
\hline LHS 2664 & FN Vir & M 4.5 & 2002-03-13 3 spectra $3600 \mathrm{~s}$ & 74 \\
\hline LHS 324 & GL Vir & M5 & $\begin{array}{l}2002-03-133 \text { spectra } 3600 \mathrm{~s} \\
2002-03-162 \text { spectra } 2400 \mathrm{~s}\end{array}$ & 72 \\
\hline LHS 36 & CN Leo & M 5.5 & $\begin{array}{l}2002-03-136 \mathrm{spectra} 7200 \mathrm{~s} \\
2002-03-144 \text { spectra } 4800 \mathrm{~s} \\
2002-03-156 \text { spectra } 7200 \mathrm{~s} \\
2002-03-166 \text { spectra } 7200 \mathrm{~s} \\
2001-01-061 \text { spectrum } 3120 \mathrm{~s}\end{array}$ & 244 \\
\hline LHS 2076 & EI Cnc & M 5.5 & $\begin{array}{l}2002-03-154 \text { spectra } 4800 \mathrm{~s} \\
2002-03-161 \text { spectrum } 1200 \mathrm{~s}\end{array}$ & 80 \\
\hline LHS 49 & Prox Cen & M 5.5 & 2001-02-02 1 spectrum $3120 \mathrm{~s}$ & 147 \\
\hline LHS 10 & UV Cet & M 5.5 & 2000-12-17 1 spectrum $3120 \mathrm{~s}$ & 109 \\
\hline LHS 248 & DX Cnc & M 6 & $2002-03-163$ spectra $3600 \mathrm{~s}$ & 17 \\
\hline LHS 2034 & $\mathrm{AZ} \mathrm{Cnc}$ & M 6 & $\begin{array}{l}2002-03-146 \text { spectra } 6000 \mathrm{~s} \\
2002-03-162 \text { spectra } 2400 \mathrm{~s}\end{array}$ & 251 \\
\hline LHS 292 & & M 6.5 & 2001-02-02 1 spectrum $3120 \mathrm{~s}$ & 103 \\
\hline LHS 429 & vB 8 & M7 & $\begin{array}{l}2002-03-133 \text { spectra } 3600 \mathrm{~s} \\
2002-03-153 \text { spectra } 3600 \mathrm{~s}\end{array}$ & 23 \\
\hline LHS 3003 & & M7 & $2002-03-143$ spectra $3600 \mathrm{~s}$ & 7 \\
\hline LHS 2397a & & M 8 & $2002-03-143$ spectra $3600 \mathrm{~s}$ & 9 \\
\hline LHS 2065 & & M9 & 2002-03-13 3 spectra $3600 \mathrm{~s}$ & 7 \\
\hline DENIS-P J104814.7-395606 & & M9 & 2002-03-14 4 spectra $4800 \mathrm{~s}$ & 4 \\
\hline DENIS-P J1058.7-1548 & & L3 & 2002-03-15 4 spectra $4800 \mathrm{~s}$ & - \\
\hline 2MASSI J1315309-264951 & & L3 & $2002-03-153$ spectra $3600 \mathrm{~s}$ & 4 \\
\hline Kelu-1 & CE 298 & L3 & $\begin{array}{l}2002-03-143 \mathrm{spectra} 3600 \mathrm{~s} \\
2002-03-163 \mathrm{spectra} 3600 \mathrm{~s}\end{array}$ & 2 \\
\hline
\end{tabular}

\section{A.8. LHS 3003, LHS 2397a, LHS 2065, vB-8, DX Cnc and DENIS-P J104814.7-395606}

Though the blue part of the spectrum is underexposed for these stars we could identify some emission lines. Moreover DENISP J104814.7-395606 showed flaring activity during the observations which led to blueshifts in the spectral lines. This star is discussed in detail by Fuhrmeister \& Schmitt (2004).

\section{A.9. DENIS-P J1058.7-1548, 2MASSI J1315309-264951 and Kelu-1}

No radial velocity correction was applied to these very latetype objects, since we had no appropriate template spectrum. Therefore the central wavelength given for these objects is not corrected for radial velocity.

For the L3 dwarf 2MASSI J1315309-264951 we report besides the detection of $\mathrm{H}_{\alpha}$ and $\mathrm{H}_{\beta}$ weak $\mathrm{Na}$ I D emission lines (see Fig. A.1). This is to our knowledge the first detection of the Na I D lines in emission in an L dwarf. These are heavily blended with airglow lines known for the UVES instrument (Hanuschik 2003). The $E W$ for $\mathrm{H}_{\alpha}$ of $24.1 \AA$ seems to imply that the brown dwarf is in a rather quiescent state since Hall (2002) found $E W$ of $121 \AA$ and of $25 \AA$ half a year later.

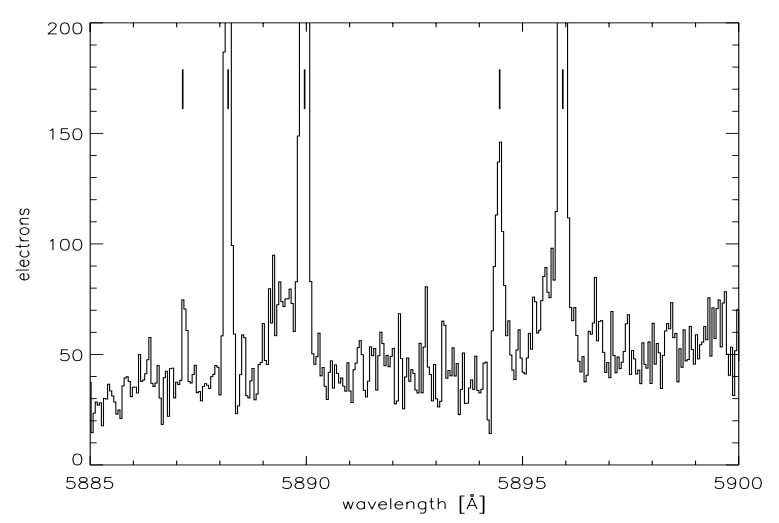

Fig. A.1. Spectrum of 2MASSI J1315309-264951 around the Na I D lines. Known airglow lines for the UVES instrument are marked with a vertical line. 